\title{
Impact of Infection Control Training Program in Improving the Quality of Healthcare
}

\author{
Aeshah N. Alqahtani, Rana H. Almaghrabi, Mohammed M. Albaadani, and Khalid Almossa
}

\section{ABSTRACT}

\begin{abstract}
The purpose of this study was to explore the impact of various training programs and guidelines on improving the healthcare quality and patient. The training programs that we have explained in this study are related to Hand Hygiene compliance, Improving clinical outcomes and reducing financial burden and Preventing healthcare associated infection, This study Applied to many health institutions and healthcare employees in the Kingdom of Saudi Arabia, It is from this analysis that improve quality of healthcare through control training program, IPC training program improve hand hygiene compliance and reduces the HAIs prevalence, improves patient outcomes and reduces healthcare costs, training of all the persons involved in primary healthcare may fill the IPC gaps.
\end{abstract}

Keywords: Infection; Quality; Healthcare; Training Programs; Saudi Arabia; hygiene.
Published Online: October 19, 2020

ISSN: $2593-8339$

DOI: $10.24018 /$ ejmed.2020.2.5.498

\section{Aeshah N. Alqahtani *}

MED, Dip IC, Infection control, Prince Sultan Military Medical City, Riyadh, Saudi Arabia.

(e-mail: An-alqahtani@ ${ }^{\circledR}$ psmmc.med.sa) Rana H. Almaghrabi

MD, MBBS, Infectious diseases consultant, Prince Sultan Military Medical City, Riyadh, Saudi Arabia. (e-mail: Ralmaghrabi@psmmc.med.sa) Mohammed M. Albaadani

BSN, RN, Prince Sultan Military Medical City, Riyadh, Saudi Arabia. (e-mail: albaadanim2@hotmail.com) Khalid Almossa

Prince Sultan Military Medical City, Riyadh, Saudi Arabia.

(e-mail: Khalidmalmousa@gmail.com)

*Corresponding Autho

\section{INTRODUCTION}

Infection prevention and control (IPC), a universally relevant component of all health systems, affects the health and safety of both, patients and healthcare providers and form an unnecessary and preventable financial burden [24]. World Health Organization (WHO) defines infection prevention and control as a scientific approach and practical solution to prevent health care associated infections (HAIs) causing unnecessary harm and at times even death, saves money, reduces the spread of antimicrobial resistance (AMR), protect patients and health workers from avoidable infections and also supports high quality, integrated, peoplecentered health services. Infection caused by bacteria, fungi, viruses or prions can affect entire body systems. Healthcare workers can play a significant role in measuring and controlling the infection by applying infection prevention and control standards. However, it demands a basic understanding of the epidemiology of diseases, risk factors, increasing susceptibility to infection, as well as the practices, procedures and treatments that may result in infections. Infection prevention and control (IPC) practices are increasingly becoming an integral part of patient care as high incidence of adverse events in healthcare institutions has brought attention to patient safety globally. However, the shortfall in its implementation contributes to the significant lack of preventing and minimizing risk/ harm to patients by minimizing them to acceptable limits [11], [29] Healthcare policies and guidelines are constantly evolving to incorporate evidence based practice reducing preventable infections in the healthcare setting. Infection prevention and control that essentially pertains to the policies, procedures, and practices to prevent transmission of healthcare associated infection (HAIs), is based on the ethical principle of no maleficence necessitating that healthcare practitioners prevent any harm [4]. The healthcare professional is obligated to reduce HAIs as much as possible if not complete elimination. Infection prevention and control is essential in any healthcare facility because Hals affect adversely not only the patients but also the healthcare professionals and visitors. The WHO estimates that one in every ten patients around the world gets an infection while receiving care, making HAIs the fourth most common cause of death [26]. The problem becomes acute during the catastrophic outbreaks likeH1NI, SARS, Ebola, MERS and SARS CoV2 outbreaks. Infection prevention and control training programs may reduce the occurrence and impact of hospital-acquired infections through educating the healthcare practitioners, patient and visitors about the best practices to prevent or control hospital acquired infections. 
The performance improvement (PI) activities need to include infection prevention program. Patient safety is one of the healthcare priorities worldwide and majority of the hospitals are engaged in activities and initiatives to provide safe health care and improve the health care outcomes, in a cost-effective manner. The training of health professionals is fundamental part of the nosocomial infection prevention program, including HAIs [12]. Unfortunately, a lack of knowledge required to control and prevent HAIs is evident among healthcare professionals [20]. The Infection control and prevention activities and initiatives should start early and be at the core of the concept of health care quality. Evidently, education of health care workers is fundamental to improve infection prevention practices [31]. Furthermore, the content and delivery of education in IPC programs needs to be standardized, with an emphasis on infection control and prevention by adhering to best practices. The education and training aspect maybe delivered by experts, but compliance needs to be ensured by local quality assurance management [5]. IPC program can play a vital role in the dissemination of knowledge and skills to ensure patient safety, adherence to safe practices and preventing risk situations. However, many hospitals lack trained infection control professionals (ICPs), even in the presence of many available opportunities to fix this gap. The diploma program in infection control established independently by Ministry of health and Saudi Commission for Health Specialties (SCFHS) along with few other institutions aims to impart required knowledge and skills to control and prevent infection in healthcare facilities. Additionally, the courses are available to prepare for American Board of infection control (CBIC), the international qualification that is the prerequisite for the accreditation standards for Joint Commission International (JCI). Current article explores the impact of various training programs and guidelines on improving the healthcare quality and patient safety.

\section{LITERATURE REVIEW AND DISCUSSION}

\section{A. Hand Hygiene compliance}

Hand hygiene is arguably the most effective and integral to the control and preventing infections in the healthcare setting. Adherence of universal precautions like hand hygiene by healthcare workers mitigates transmission of microorganisms and eventual HAIs [19]. However, low compliance with easy to follow infection control measures has proven to be a bottleneck [28]. Early studies have shown that the hands of the healthcare workers transmit microorganisms from contaminated surfaces and objects to patient as well as spread among them [27]. Optimized hand hygiene compliance is among the core impacts of the IPC training program. Well-structured and implemented infection prevention and control program can improve hand hygiene compliance by up to $80 \%$, which in turn reduced the occurrence of HAls by up to 50\% [17]. Early evidence indicates that a strict compliance with the hand hygiene regimen can prevent more than half of the HAIs incidence. A simple hand hygiene compliance can reduce half of over 6 million cases of hospital-acquired infections globally every year which in turn can prevent large number of adverse clinical outcomes, and morbidity and mortality among patient and healthcare practitioners [16]. Alarmingly, hand hygiene compliance is often low and overlooked warranting its promotion in every healthcare institution. An early study showed lower than $50 \%$ compliance with hand hygiene regulations globally [27]. Quite fittingly, as many as $60 \%$ fewer case of healthcare-associated infections was found in healthcare institutions where hand hygiene practice was considered a priority as compared to those not having proper hand hygiene practice. Quality assurance (QA) department may devise and implement the hand hygiene regulations as part of the wider infection prevention and control program. Furthermore, QA may also take initiative in promotion and education activities about hand hygiene by designing and strategic placement of hand hygiene promotional posters. Installation of hand hygiene kits at strategic places like around the patient wards, operation theatres, and ICU units may also be undertaken. It is worthwhile to note that the teaching of hand hygiene has assumed a central and sometimes sole position as a method of promoting patient safety by preventing HAIs [25], [10], [30]. The WHO recommend hand hygiene as an important patient safety goal; however, good practices should not be restricted to a single set of procedures or technique.

\section{B. Improving clinical outcomes and reducing financial burden}

Although principles, pathogenesis and transmission mechanisms of HAIs, are taught as part of medical courses, infection prevention and control (IPC) as the hallmark of quality and safety in patient care is largely unexplored, globally [3], [2], [23] and does not have the same weightage as developing clinical skills, like diagnosis and treatment [5]. Well-designed and implemented IPC training programs significantly improve the clinical outcome in a cost-effective manner. HAIs significantly increase the morbidity, mortality, and economic cost [24], contributing to between $3.18 \%$ and $10.73 \%$ of hospital admissions and readmissions adversely impacting patient's quality of life in Saudi Arabia [1]. This is a substantial rate considering that more than one third of these adverse events are preventable [1]. An effective infection prevention and control training program can reduce nosocomial infections by at least $30 \%$ significantly improving clinical outcome [18]. These programs have been shown to be effective in controlling infection outbreaks in the healthcare setting and the quality assurance departments across Saudi Arabia may evolve measures and mechanisms enhancing the efficacy of infection prevention and control training programs and subsequent compliance [1]. This mainly entails the sufficient provision of personal protective equipment like gloves, gowns, soaps and where necessary, mask, boots, and other infection control supplies such as chlorine and disposable rags.

The IPC training programs, if well designed and implemented, may also reduce the healthcare related costs both for the patient and the healthcare institution. Early study has shown that HAIs cost $\$ 5$ billion the U.S. healthcare systemin 2015 [6]. UK, another country with an 
excellent healthcare system, suffered an HAIs associated loss of $£ 930$ million [34], [35], [13]. These are, mostly, the superficial estimates related to the demonstrable direct cost; the indirect costs associated with HAIs are too large to fathom. To an individual, an effective IPC training program may reduce the costs associated with a prolonged stay in the hospital and even the loss of an employment opportunity and wages. There are also indirect costs such as the family members' time lost from work or other activities to care the affected individual. To the healthcare institution, effective IPC programs may considerably reduce the cost associated with readmissions, lengthy stay for patients, unwarranted increased use of antimicrobials, and even the incremental costs associated with the surveillance and isolation measures for Antibiotic Resistant Organisms (AROs) [26]. Other activities related to HAIs that exacerbate the operational Costs include the costs attributable to containing outbreaks and even diagnosing and managing HAIs [13]. Effective IPC training program may significantly reduce the prevalence of HAIs and related economic costs.

\section{Preventing healthcare associated infection}

Well-designed evidence- based IPC training programs may significantly reduce the incidence of HAIs in the healthcare facilities as much as $65 \%$ [18]. It becomes more important in the event of an outbreak like COVID19 during which the healthcare workers are disproportionately affected as demonstrated during 2015 Ebola outbreak in Guinea. The IPC training program is evaluated using IPC score of healthcare facilities reflecting the number of IPC trained staff. The healthcare facilities with the higher number of staff trained in infection prevention and control had fewer instances of HAIs as compared to institutions not having IPC trained personnel. Healthcare facilities with one and two IPC trained individual were four and ten times, respectively likely to have an IPC score above the median as compared to those with no IPC trained staff [16]. Quality assurance department can play an important role in implementing IPC training to the medical staff. Accumulating evidence has shown that even a slight increase in the number of IPC trained staff may significantly reduce the incidence of HAIs in the healthcare setting. Online training program enabling participation of large number of health professionals and the feedback with information on local hospital HAIs rates can be valuable.

Early research show that clinical skills and performance are directly correlated with the teaching resources and the technological tools used [7], [33] like software [15], on-line modules [7], [32] and simulated scenarios [22]. Simulations provides control, manipulation and reproduction of rare, complex or high-risk scenarios for patients and students making it safer than real life scenario yet developing the required skills [14], [21].

Trained professionals need to be supported and encouraged at local level to act as a role model and to transfer knowledge to their peers likely improving adherence to infection with prevention and control measures [36]. Early study showed better compliance with infection prevention and control policies (like hand hygiene and personal protective equipment) in the presence of effective local leadership [9]. Furthermore, interdisciplinary cooperation between professionals from different disciplines at a healthcare setting also influences attitude towards IPC guidelines [8].

\section{CONCLUSION}

An alarmingly high incidence of healthcare associated infection globally warrants an effective intervention that can reduce morbidity, mortality, and the healthcare related costs for individual and the facilities. Effective IPC training program significantly improve hand hygiene compliance, which is the most effective measure for preventing HAIs. Additionally, it reduces the HAIs prevalence, improves patient outcomes, and reduces healthcare costs related to HAIs. The quality assurance department may fill the IPC gaps through sufficient training of all the persons involved in primary healthcare. It may also provide sufficient PPE, standardize IPC protocols, implement contemporary IPC practices, followed with sufficient monitoring and supervision to ensure compliance. IPC program focused on safety culture, communication and teamwork should be interdisciplinary and innovative coupled with self-evaluation and self-criticism. Such programs not only train the healthcare professionals but also make them aware of patient safety issues, directly influencing the quality and safety of the care provided.

\section{RECOMMENDATIONS}

As a result of our research and upon on the literature review, the following are our recommendations to be taken into considerations in the future:

-It is recommended that to train the managers and entrepreneurs the employees in health facilities on Infection Control Training Program to be aware of harms of Infection, train human recourses professionals and all employees about them, prevent and get rid of some unhygienic behaviors in their organizations.

-A policy against Infection and Control Training Program ought to be adopted altogether of health facilities to observe this development. Infection rates could also be reduced through employee's development and establishing a system for observance these Phenomenon to all healthcare facilities.

- It is recommended that to improve the Quality of Healthcare through make and improve the Infection Control Training Program.

-It is recommended that to explore the fact of quality of healthcare further in Saudi Arabia and countries of similar cultural backgrounds.

\section{ACKNOWLEDGMENT}

The authors have a deep gratitude towards Prince Sultan Military Medical City for sponsoring this work and supporting the aims of this research paper. The author is also grateful to employees in Prince Sultan Military Medical City, Saudi Arabia for their help in performing the irradiation. 


\section{REFERENCES}

[1] Assiri, A., Choudhry, A., Alsaleh, S., Alanazi, K. and Alsaleh, S 2014. Evaluation of Infection Prevention and Control Programmes (IPC), and Assessment Tools for IPC-Programmes at MOH-Health Facilities in Saudi Arabia. Open Journal of Nursing, 483-492.

[2] Bohomol, E. \& Cunha, I. C. 2015. Teaching patient safety in the medical undergraduate program at the Universidade Federal de Sao Paulo. Einstein (Sao Paulo), 13, 7-13

[3] Botene, D. Z. \& Pedro, E. N. 2014. [Health professionals and hand hygiene: a question of pediatric patient safety]. Rev Gaucha Enferm, $35,124-9$.

[4] Cabral, J. \& AG, R. 2019. Blue Light Disinfection in Hospital Infection Control: Advantages, Drawbacks, and Pitfalls. Antibiotics (Basel), 8.

[5] Carrico, R. M., Garrett, H., Balcom, D. \& Glowicz, J. B. 2018 Infection Prevention and Control Core Practices: A Roadmap for Nursing Practice. Nursing, 48, 28-29.

[6] Castro-Sanchez, E. \& Holmes, A. H. 2015. Impact of organizations on healthcare-associated infections. J Hosp Infect, 89, 346-50.

[7] D, P. W., C, J. P., B, E. M. \& Smith, M. K. 2011. An interdisciplinary infection control education intervention: necessary but not sufficient J Grad Med Educ, 3, 203-10.

[8] Damschroder, L. J., Banaszak-Holl, J., Kowalski, C. P., Forman, J., Saint, S. \& Krein, S. L. 2009. The role of the champion in infection prevention: results from a multisite qualitative study. Qual Saf Health Care, 18, 434-40

[9] De Bono, S., Heling, G. \& Borg, M. A. 2014. Organizational culture and its implications for infection prevention and control in healthcare institutions. J Hosp Infect, 86, 1-6.

[10] dogra, S., Mahajan, R., Jad, B. \& Mahajan, B. 2015. Educationa interventions to improve knowledge and skills of interns towards prevention and control of hospital-associated infections. Int J Appl Basic Med Res, 5, S54-7.

[11] Duarte SDA, C., Stipp, M. A., Da Silva, M. M. \& De Oliveira, F. T 2015. Adverse events and safety in nursing care. Rev Bras Enferm, 68, 136-46, 144-54.

[12] Fayerberg, E., Bouchard, J. \& Kellie, S. M. 2013. Knowledge, attitudes and practice regarding Clostridium difficile: a survey of physicians in an academic medical center. Am J Infect Control, 41, 266-9.

[13] Haque, M., Sartelli, M., Mckimm, J. \& Abu Bakar, M. 2018. Health care-associated infections - an overview. Infect Drug Resist, 11, 2321-2333.

[14] Holland, R., Meyers, D., Hildebrand, C., Bridges, A. J., Roach, M. A. \& Vogelman, B. 2010. Creating champions for health care quality and safety. Am J Med Qual, 25, 102-8.

[15] Huang, Y., Xie, W., Zeng, J., Law, F. \& Ba-Thein, W. 2013. Limited knowledge and practice of Chinese medical students regarding health care associated infections. J Infect Dev Ctries, 7, 144-51.

[16] Ibeneme, S., Maduako, V., Ibeneme, G. C., Ezuma, A., Ettu, T. U., Onyemelukwe, N. F., Limaye, D. \& Fortwengel, G. 2017. Hand Hygiene Practices and Microbial Investigation of Hand Contact Swab among Physiotherapists in an Ebola Endemic Region: Implications for Public Health. Biomed Res Int, 2017, 5841805.

[17] Kingston, L. M., O'connell, N. H. \& Dunne, C. P. 2018. A comparative study of hand hygiene and alcohol-based hand rub use among Irish nursing and medical students. Nurse Educ Today, 63, 112-118.

[18] Koo, E., Mcnamara, S., Lansing, B., Olmsted, R. N., Rye, R. A. Fitzgerald, T. \& Mody, L. 2016. Making infection prevention education interactive can enhance knowledge and improve outcomes: Results from the Targeted Infection Prevention (TIP) Study. Am J Infect Control, 44, 1241-1246.

[19] Luangasanatip, N., Hongsuwan, M., Limmathurotsakul, D., Lubell, Y., Lee, A. S., Harbarth, S., Day, N. P., Graves, N. \& Cooper, B. S 2015. Comparative efficacy of interventions to promote hand hygiene in hospital: systematic review and network meta-analysis. BMJ, 351 h3728.

[20] Mavros, M. N., Alexiou, V. G., Vardakas, K. Z., Tsokali, K., Sardi, T. A. \& Falagas, M. E. 2012. Underestimation of Clostridium difficile infection among clinicians: an international survey. Eur $\mathrm{J}$ Clin Microbiol Infect Dis, 31, 2439-44.

[21] Morello, R. T., Lowthian, J. A., Barker, A. L., Mcginnes, R., Dunt, D. \& Brand, C. 2013. Strategies for improving patient safety culture in hospitals: a systematic review. BMJ Qual Saf, 22, 11-8.

[22] Nguyen, L. K. N., Megiddo, I. \& Howick, S. 2019. Simulation model for transmission of health care-associated infection: A systematic review. Am J Infect Control.
[23] O'brien, D., Richards, J., Walton, K. E., Phillips, M. G. \& Humphreys, H. 2009. Survey of teaching/learning of healthcareassociated infections in UK and Irish medical schools. J Hosp Infect, 73, 171-5.

[24] Pittet, D. \& Donaldson, L. 2005. Clean Care is Safer Care: a worldwide priority. Lancet, 366, 1246-7.

[25] Shekhter, I., Nevo, I., Fitzpatrick, M., Everett-Thomas, R., Sanko, J S. \& Birnbach, D. J. 2009. Creating a common patient safety denominator: the interns' course. J Grad Med Educ, 1, 269-72.

[26] Storr, J., Twyman, A., Zingg, W., Damani, N., Kilpatrick, C., Reilly, J., Price, L., Egger, M., Grayson, M. L., Kelley, E. \& Allegranzi, B 2017. Core components for effective infection prevention and control programmes: new WHO evidence-based recommendations. Antimicrob Resist Infect Control, 6, 6 .

[27] Treise, D., Weigold, M. F., Birnbrauer, K. \& Schain, D. 2016. The best of intentions: patients' intentions to request health care workers cleanse hands before examinations. Health Commun, 31, 425-33.

[28] Tromp, M., Huis, A., De Guchteneire, I., Van Der Meer, J., Van Achterberg, T., Hulscher, M. \& Bleeker-Rovers, C. 2012. The shortterm and long-term effectiveness of a multidisciplinary hand hygiene improvement program. Am J Infect Control, 40, 732-6.

[29] Van Rosse, F., De Bruijne, M., Suurmond, J., Essink-Bot, M. L. \& Wagner, C. 2016. Language barriers and patient safety risks in hospital care. A mixed methods study. Int J Nurs Stud, 54, 45-53.

[30] Varkey, P., Karlapudi, S., Rose, S. \& Swensen, S. 2009. A patient safety curriculum for graduate medical education: results from a needs assessment of educators and patient safety experts. Am J Med Qual, 24, 214-21.

[31] Ward, D. J. 2011. The role of education in the prevention and control of infection: a review of the literature. Nurse Educ Today, 31, 9-17.

[32] Wetzel, A. P., Dow, A. W. \& Mazmanian, P. E. 2012. Patient safety attitudes and behaviors of graduating medical students. Eval Health Prof, 35, 221-38.

[33] Wilson, A. R., Fabri, P. J. \& Wolfson, J. 2012. Human error and patient safety: interdisciplinary course. Teach Learn Med, 24, 18-25.

[34] Wistrand, C., Falk-Brynhildsen, K. \& Nilsson, U. 2018. National Survey of Operating Room Nurses' Aseptic Techniques and Interventions for Patient Preparation to Reduce Surgical Site Infections. Surg Infect (Larchmt), 19, 438-445.

[35] Woldegioris, T., Bantie, G. \& Getachew, H. 2019. Nurses' Knowledge and Practice Regarding Prevention of Surgical Site Infection in Bahir Dar, Northwest Ethiopia. Surg Infect (Larchmt), 20, 71-77.

[36] Zingg, W., Holmes, A., Dettenkofer, M., Goetting, T., Secci, F., Clack, L., Allegranzi, B., Magiorakos, A. P. \& Pittet, D. 2015 Hospital organisation, management, and structure for prevention of health-care-associated infection: a systematic review and expert consensus. Lancet Infect Dis, 15, 212-24.

Aeshah N. Alqahtani was born in Saudi Arabia and stayed there. He completed his undergraduate degree and his master degree in Saudi Arabia, $\mathrm{He}$ is interested in the field of Infection control and healthcare research, Being raised in a family who is known for their passion for learning has enhanced his personal values and attitudes towards medicine. As a matter of fact, he has been always motivated to peruse his education and research and be the best person. After accomplishing that he had a chance to work as a lecturer and researcher at many Saudi universities and as MED, DipIC in Prince Sultan Military Medical City, Riyadh, Saudi Arabia.

Rana H. Almaghrabi was born in Saudi Arabia and stayed there. He completed his undergraduate degree and his master degree in Saudi Arabia, $\mathrm{He}$ is interested in the field of Infection control and healthcare research After accomplishing that he had a chance to work as a lecturer and researcher at many Saudi universities and as MD, MBBS and Infectious diseases consultant in Prince Sultan Military Medical City, Riyadh, Saudi Arabia.

Mohammed M. Albaadani was born in Saudi Arabia and stayed there He completed his undergraduate degree and his master degree in Saudi Arabia, and had a chance to work as BSN, RN in Prince Sultan Military Medical City, Riyadh, Saudi Arabia.

Khalid Almossa was born in Saudi Arabia and stayed there. He completed his undergraduate degree and his master degree in Saudi Arabia, and had a chance to work as Doctor in Prince Sultan Military Medical City, Riyadh, Saudi Arabia. 